\title{
Effect of Nasal Calcitonin on the Health-Related Quality of Life in Postmenopause Women Affected With Low Bone Density
}

\author{
Majid Shohrati ${ }^{1}$; Noushin Bayat ${ }^{2,}$; Amin Saburi ${ }^{3}$; Zahra Abbasi ${ }^{4}$ \\ ${ }^{1}$ Chemical Injuries Research Center, Baqiyatallah University of Medical Sciences, Tehran, IR Iran \\ ${ }^{2}$ Internal Diseases Department, Faculty of Medicine, Baqiyatallah University of Medical Sciences, Tehran, IR Iran \\ ${ }_{3}^{3}$ Radiology Department, Birjand Atherosclerosis and Coronary Artery Research Center, Birjand University of Medical Sciences, Birjand, IR Iran \\ ${ }^{4}$ Pharmaceutical Sciences Branch, Islamic Azad University (IAUPS), Tehran, IR Iran \\ ${ }^{*}$ Corresponding Author: Noushin Bayat, Internal Diseases Department, Faculty of Medicine, Baqiyatallah University of Medical Sciences, Tehran, IR Iran. Tel: +98-2188600067, \\ E-mail: md.researcher@yahoo.com
}

Received: June 1, 2012; Revised: December 5, 2014; Accepted: March 6, 2015

\begin{abstract}
Background: Physical activity and mental health could be affected by osteoporosis and various therapeutic options such as calcitonin may influence Quality Of Life (QOL) of these patients with Low Bone Density (LBD).

Objectives: This study aimed to evaluate the effect of nasal calcitonin on QOL in post menopause women with LBD.

Patients and Methods: This clinical trial study was performed on one hundred and fifteen menopause women with LBD less than 1 SD in Bone Mineral Densitometry (BMD) referred to Baqiyatallah Hospital in Tehran, Iran, during 2009 - 2010. They were assigned to receive 200 IU calcitonin nasal spray along with calcium $(1000 \mathrm{mg}$ ) and vitamin D (400 IU) for 6 months. Quality of life was assessed by Short-Form 36 (SF-36) questionnaire (Persian-validated version).

Results: The mean age $( \pm$ SD) of the participants was $58.75 \pm 8.15$ years. Intranasal spray of calcitonin increased QOL scores significantly $(88.05 \pm 15.63$ vs. $92.15 \pm 13.22$, P value $=0.000)$. Bonemineral density of spine was increased from $0.834 \pm 0.11$ to $0.12 \pm 0.852$ and this difference in BMD of lumbar spine was statistically significant ( $P$ value: 0.003 ) but not significant in femur's BMD (P value $=0.061)$. In comparison with BMD indexes, The QOL scores especially Mental Health domain changes had only a significant correlation with the changes of total T score in $\mathrm{BMD}(\mathrm{P}=0.031$, Coefficient Correlation $=0.248)$.

Conclusions: It seems that nasal spray of calcitonin can effectively improve QOL of women with LBD and QOL changes were not influenced by clinical or para-clinical alteration. Mental health domain must be more considered in further studies as a predicting domain for HealthRelated Quality of Life(HR-QOL) changes.
\end{abstract}

Keywords: Osteoporosis; Oteoporosis; Postmenopausal; Quality of Life, Calcitonin; Bone Density

\section{Background}

Bone-density depleting disorders including Osteoporosis (OPS) and Osteopenia (OPN) are age-related disorders characterized by Low Bone Mineral Density (LBMD) and destruction of bone tissue micro-architecture, resulting in susceptibility to the pathologic fracture (1). Osteoporosis and OPN are commonly expected in postmenopause women over fifty years of age because of hormonal imbalance. This hormonal imbalance could induce bone-tissue resorption versus reconstruction. Bone micro-architecture remodeling is continuous and natural process containing bone resorption and new bone formation. These regular processes also control the calcium homeostasis, and replacement of bone tissue affected by microfractures (2). Therefore, OPS can cause several major skeletal problems, such as pathologic fracture and thereby put at risk the general health.

Osteoporotic fractures (vertebral or hip) as pathologic fractures are associated with significant mortality and morbidity. Osteoporotic individuals may have severe and intractable pain caused by vertebral fractures. These complications can affect the Health-Related Quality of Life (HR-QOL). Previous studies showed that HR-QOL (estimated by the SF-36 questionnaire) was affected two years after vertebral and hip fractures (3).

Low Bone Density (LBD) is one of the most challenging worldwide therapeutic problems, particularly in the developed country $(2,4)$. Different therapeutic agents have been recommended for OPS and OPN that they purpose to prevent the disease progression or reduce the risk of fractures. Calcitonin is an antiresorptive agent, which has been confirmed for the treatment of OPS since 2 decades ago (5-8). Calcitonin (nasal or injection form) via inhibiting the osteoclastic activity can reduce the risk of osteoporotic fracture.

\section{Objectives}

Previously, the therapeutic effects of calcitonin on OPS and LBD were investigated by using BMD (9). This study aimed to evaluate the effect of nasal calcitonin on QOL in postmenopause women with LBD.

Copyright ( 2015, Iranian Red Crescent Medical Journal. This is an open-access article distributed under the terms of the Creative Commons Attribution-NonCommercial 4.0 International License (http://creativecommons.org/licenses/by-nc/4.0/) which permits copy and redistribute the material just in noncommercial usages, provided the original work is properly cited. 


\section{Patients and Methods}

A total of 150 women were enrolled in the study. We evaluated postmenopause women who had referred to the general clinic of rheumatology in Baqiyatallah University of Medical Sciences, Tehran, Iran during a 1-year period between 2009 and 2010. Finally, one hundred and fifteen cases met the inclusion criteria: (1) BMD at any site of measurement with a T score $<-1$ SD which is accepted as an international criterion of LBD (osteopenia) in postmenopause women; (2) no evidence for secondary OPN; (3) no sufficient response to the pharmacological doses of vitamin D within the past 6 months. Patients were overruled if they had present or previous history of nasal polyps or a nasal septal perforation and previous treatment episode with prednisone or other medication which interfering bone metabolism such as estrogen or progesterone, bisphosphonates, and calcitonin.

The presence of secondary OPN, other bone disorders or other important medical conditions such as hyperparathyroidism, hyperthyroidism, neoplastic disorders and other were left out based on the patient's history, whole body physical examination, routine laboratory tests, and, if necessary, hormone assessment and additional assessment for suspected patients. The Baqiyatallah university ethics committee had approved the study plan. All participants filled out the informed consent to take part in the study. Patient's compliance was considered throughout the study by phone call.

The patients received 200 IU nasal calcitonin with 2 word brand names (Nasocalcin nasal spray 200 IU, Sinadaru, Tehran, IR Iran and Miacalcic® 200 IU nasal spray, Novartis, UK) once a day along with calcium tablet $(1000 \mathrm{mg})$ and vitamin D (400 IU) for 6 months. The suspected adverse effects were explained for every participant and all patients were visited every 2 months for adverse effect and efficacy of treatment on clinical manifestation.

Also, BMD was evaluated by a dual-energy X-ray absorptiometry (Norland Company, USA) at the femoral neck and lumbar spine (L2 - L4). Bone mineral density was assessed at the end of the study on last visit and compared with the baseline.

Urine and blood samples were collected for the bio- chemical bone marker assessment at the first and 6 months after the beginning of the treatment. Calcium homeostasis was observed by measuring serum and 24-hour urinary calcium and phosphorus levels, Parathormone (PTH) serum levels and serum Alkaline Phosphatase (ALP). Other laboratory parameters, which were assessed during the treatment, were as follow: cell blood count, serum calcium, serum phosphorus, creatinine (Cr), albumin, Blood Urea Nitrogen (BUN), electrolyte (Na and $\mathrm{K}$ ) and liver aminotransferases. Serum and urine samples were collected on the morning without fasting and avoidance from tobacco. At this clinical survey, a detailed HR-QOL questionnaire including the validated Persian version of Short-Form 36 (SF-36) questionnaire was used (10).

We used the Persian version of the SF-36 questionnaire which was extensively evaluated in both reliability and validity base on Persian conditions (10-12). Also, the SF36 has been confirmed under Swedish conditions and in several other countries (13-15).

Moreover, the Quantitative variables' distribution was assessed using the Kolmogorov-Smirnov test and then represented as Mean \pm SD (Standard Deviation) and qualitative variables' frequency is presented as a percentage. The paired t-test and Pearson correlation were used to compare the impact of calcitonin on the quantitative variables. Also, the Mantel-Hanzel and two-tailed Wilcoxon tests were used to compare between qualitative variables and nonparametric variables. Finally, linear regression model was used to justify the effect of the confounding factors. SPSS version 16 (Illinois, USA) was used to analyze the data and $\mathrm{P}$ values $<0.05$ were considered statistically significant.

\section{Results}

One hundred and fifteen postmenopause women were included (age range, 47 - 84 years). All patients completed the two steps of the study (failure to follow: $0 \%$ ). There were no individuals who reported considerable adverse reactions during the investigation. Table 1 shows the baseline characteristics of the study subjects. This difference in BMD of lumbar spine was statistically significant but not significant in femur's BMD.

\begin{tabular}{|c|c|c|c|}
\hline & Baseline & 6 Mon & $\mathbf{P}$ \\
\hline Age, $y$ & $58.75 \pm 8.15$ & & \\
\hline The mean age at menopause & $47.11 \pm 5.43$ & & \\
\hline Mean year beyond menopause & $12.32 \pm 8.70$ & & \\
\hline BMI, $\mathrm{kg} / \mathrm{m}^{2}$ & $28 \pm 4.10$ & & \\
\hline Baseline weight, kg & $67.52 \pm 5.47$ & & \\
\hline BMD (femoral neck ), $\mathrm{g} / \mathrm{cm}^{2}$ & $0.699 \pm 0.104$ & $0.708 \pm 0.100$ & 0.061 \\
\hline BMD (lumbar spine), $\mathrm{g} / \mathrm{cm}^{2}$ & $0.834 \pm 0.112$ & $0.852 \pm 0.122$ & 0.003 \\
\hline
\end{tabular}

\footnotetext{
a Abbreviations: BMI, body mass index; BMD, bone mineral density.
} 
C-telopeptide of type I collagen (CTX; as a bone resorptive marker) over the 6-month treatment was decreased significantly $(3.203 \pm 2.24$ vs. $2.497 \pm 1.657$, P value $<0.001)$. At the baseline, $55.3 \%$ of the patients suffered from low back pain although the frequency of the low back pain during the study changed to $3.5 \%, 1.8 \%$ and $0 \%$, which is statistically significant $(\mathrm{P}<0.001)$.

The 24-hour urine Ca level was increased significantly compared to the baseline (from $216.96 \pm 114.68$ to 270.87 $\pm 155.47, \mathrm{P}<0.001)$. Changes in other biochemical serum markers were not significant $(\mathrm{P}>0.050)$. The 24-hour urine $\mathrm{Cr}$ level raised from $0.93 \pm 0.35$ to $1.08 \pm 0.37$ that this change was significant. Moreover, BUN at the baseline was $17.08 \pm 4.07$ and the amount was significantly increased at the end of the study to $18.17 \pm 4.35$ ( $\mathrm{P}=$ 0.043). Serum levels of PTH, Ca, AST, ALT and ALP total were not changed significantly ( $P$ value $>0.050)$. Also, the changes in femur BMD amount were significantly correlated with weight $(\mathrm{P}=0.014, \mathrm{r}=-0.244)$ and $\mathrm{BMI}(\mathrm{P}$ $=0.022, \mathrm{r}=-0.301$ ).

At baseline measurement, the total QOL score were $88.05 \pm 15.63$, and also 6 months after the treatment the SF36 score was $92.15 \pm 13.22$. This difference in HR-QOL score was statistically significant $(\mathrm{P}<0.001)$ (Table 2 ). By using partial correlation, although the probable confounding role of age, age at menopause number of pregnancy, the amount of exercise per day and BMI were adjusted, the difference between baseline and final total score of HR-QOL was statistically significant $(P<0.001)$. Regarding to the univariate test and by using the multivariate analysis, we adjusted the HR-QOL score changes regarding to the role of demographic variables. Also, changes in the HR-QOL score had a significant correlation only with educational level $(\mathrm{P}=0.010, \mathrm{~F}=4.252$, Power $=97.5 \%)$; however, other demographic characteristics such as age at menopause, age, BMI, number of pregnancy and the amount of exercise per day had no significant correlation with HR-QOL $(\mathrm{P}>0.050)$.
Changes in HR-QOL domains over the 6-month treatment period are presented in Table 3. Changes in HR-QOL domains only in General Health (GH) and the short form 36 Health survey (SF-36) were not statistically significant (0.309 and 0.917, respectively). By using the linear regression model, all of the HR-QOL's domains changed significantly compared to the changes in total HR-QOL score $(\mathrm{P}<0.001)$ and also affected by other domains. Table 4 demonstrates the median change in SF-36 score over follow-up for each domain according to its correlation with together and it seems that Mental Health (MH) was the most effective item, which affected the total SF-36 score change $(\mathrm{P}<0.001, \mathrm{t}=26.13)$.

Table 2. Dimensions of the Short-Form 36 Health Survey Questionnaire

\begin{tabular}{|lc}
\hline Area/Dimension & No of Questions \\
\hline Functional status & 10 \\
\hline Physical functioning & 2 \\
\hline Social functioning & 4 \\
\hline Role limitations (physical problems) & 3 \\
\hline Role limitations (emotional problems) & \\
\hline Wellbeing & 5 \\
\hline Mental health & 4 \\
\hline Vitality & 2 \\
\hline Pain & \\
\hline Overall evaluation of health & 5 \\
\hline General health perception & 1 \\
\hline Health change & 36 \\
\hline Total
\end{tabular}

Table 3. Changes in Health-Related Quality of Life Domains During the Study

\begin{tabular}{lccc}
\hline Domains & Before & After & PValue \\
\hline Total score & $88.05 \pm 15.63$ & $92.15 \pm 13.22$ & $0.000^{\mathrm{a}}$ \\
\hline Physical function & $21.79 \pm 4.38$ & $22.82 \pm 3.77$ & $0.000^{\mathrm{a}}$ \\
\hline Role physical & $6.05 \pm 1.47$ & $6.28 \pm 1.43$ & $0.004^{\mathrm{a}}$ \\
\hline Bodily pain & $6.00 \pm 2.06$ & $6.78 \pm 1.55$ & $0.000^{\mathrm{a}}$ \\
\hline General health & $7.45 \pm 1.65$ & $7.51 \pm 1.46$ & 0.309 \\
\hline Social function & $7.21 \pm 1.72$ & $7.21 \pm 1.26$ & 0.917 \\
\hline Role emotional & $4.56 \pm 1.29$ & $4.77 \pm 1.17$ & $0.001^{\mathrm{a}}$ \\
\hline Vitality & $13.55 \pm 3.99$ & $14.06 \pm 3.66$ & $0.002^{\mathrm{a}}$ \\
\hline Mental health & $18.96 \pm 4.61$ & $19.73 \pm 3.59$ & $0.000^{\mathrm{a}}$ \\
\hline
\end{tabular}

\footnotetext{
a Statistically significant.
} 
Table 4. Linear Regression Model for the Correlation Among the Changes in Health-Related Quality of Life Domains During the Study a

\begin{tabular}{|c|c|c|c|c|c|c|c|c|c|}
\hline & PF & $\mathbf{R P}$ & BP & GH & SF & ER & Vitality & MH & $\mathbf{t}$ \\
\hline PF & & & & & & & & & 25.61 \\
\hline $\mathrm{r}$ & 1 & & & & & & & & \\
\hline $\mathrm{P}$ & & & & & & & & & \\
\hline RP & & & & & & & & & 7.78 \\
\hline $\mathrm{r}$ & 0.388 & 1 & & & & & & & \\
\hline $\mathrm{P}$ & $0.000^{b}$ & & & & & & & & \\
\hline BP & & & & & & & & & 13.20 \\
\hline $\mathrm{r}$ & 0.287 & 0.212 & 1 & & & & & & \\
\hline $\mathrm{P}$ & $0.002^{b}$ & $0.024^{b}$ & & & & & & & \\
\hline GH & & & & & & & & & 11.28 \\
\hline $\mathrm{r}$ & 0.118 & 0.165 & 0.117 & 1 & & & & & \\
\hline $\mathrm{P}$ & 0.211 & 0.080 & 0.216 & & & & & & \\
\hline SF & & & & & & & & & 10.05 \\
\hline $\mathrm{r}$ & 0.117 & 0.037 & 0.294 & -.014 & 1 & & & & \\
\hline $\mathrm{P}$ & 0.216 & 0.697 & $0.002^{b}$ & 0.879 & & & & & \\
\hline ER & & & & & & & & & 9.81 \\
\hline $\mathrm{r}$ & 0.134 & 0.394 & 0.014 & 0.192 & 0.162 & 1 & & & \\
\hline$P$ & 0.155 & $0.000^{b}$ & 0.886 & $0.041^{b}$ & 0.086 & & & & \\
\hline Vitality & & & & & & & & & 22.01 \\
\hline $\mathrm{r}$ & -0.007 & 0.008 & 0.074 & 0.036 & 0.182 & 0.074 & 1 & & \\
\hline $\mathrm{P}$ & 0.943 & 0.936 & 0.437 & 0.705 & 0.053 & 0.434 & & & \\
\hline MH & & & & & & & & & 26.13 \\
\hline $\mathrm{r}$ & 0.201 & 0.178 & 0.054 & 0.167 & 0.282 & 0.267 & 0.415 & 1 & \\
\hline$P$ & $0.032^{b}$ & 0.059 & 0.565 & 0.076 & $0.002^{b}$ & $0.004^{b}$ & $0.000^{b}$ & & \\
\hline
\end{tabular}

a Abbreviations: BP, bodily pain; GH, general health; $\mathrm{MH}$, mental health; PF, physical function; ER, Emotional Role functioning; P, P value; r, correlation coefficient; RP, physical role functioning; $\mathrm{SF}$, social function; $\mathrm{VT}$, vitality.

b Statistically significant.

The changes in HR-QOL domains score during the study were assessed according to the BMD changes and we found that only T-score of spine's BMD and total T-score's changes were significantly correlated with the HR-QOL total score changes $(\mathrm{P}=0.003$ and $\mathrm{P}=0.028$, respectively). By using the multivariate analysis, we adjusted the changes in the score of HR-QOL domains regarding to the role of demographic variables and we found that only changes in $\mathrm{MH}$ score were significantly correlated with total BMD score changes $(\mathrm{P}=0.037)$, BP score was significantly correlated with BMI $(\mathrm{P}=0.049)$ and RP score was also significantly correlated with BMI and age at menopause $(\mathrm{P}=0.009$ and $\mathrm{P}=0.013$, respectively).

\section{Discussion}

Osteoporosis and the decrease in BMD is a major health problem throughout the world and its prevalence is increasing especially in developed countries because of increasing in the community age (16). Osteoporosis is the main cause of pathologic hip and vertebral fractures in elderly (17). Also, OPS and decrease in bone mass can increase mortality and morbidity rate substantially $(1,17$, 18). Previous study demonstrated that the osteoporotic cases have lower QOL due to limitation in physical activity, chronic mild pain and etc. In spite of the comorbidity and fracture, individuals who affected by lower bone density had poorer QOL (19). The preventive, analgesic and therapeutic impact of nasal calcitonin on the spinal fracture and therefore back pain in the osteoporosis was declared (20). Also, QOL improvement (regarding to the improvement in spine BMD) was coordinated by significant reduction in the frequency of low back pain, which is match with previous reports in osteoporotic patients without the obvious bone fracture (21). Therefore, it is expected that an effective treatment protocol can improve the HR-QOL of these patients.

Calcitonin is bone resorptive inhibitors, which has approved for treatment of osteoporosis, low bone density 
and other disorder involving increased bone turnover since 25 years ago. The present study is the first from Iran to assess the effectiveness of nasal calcitonin on HR-QOL in osteopenic postmenopause women. However, the present study showed a raise in spine BMD more than femoral BMD that this is similar to previous trial studies. Also, BMD by Dual-energy X-ray absorptiometry (DXA) is a reliable method for tracking the response to treatment in patients with OPS. Changes in BMD score when appear that the bone density be increased at least 3 - 5\% from the baseline (22). Alike previous study, in the present study, spinal BMD altered significantly. Trovas et al. confirmed the efficacy of 200 IU daily intranasal calcitonin on improvement of lumbar BMD (7).

However, we found that changes in the spine T-score were correlated with changes in the total HR-QOL score, especially $\mathrm{MH}$. In addition, patients with higher grades of spinal bone density loss showed lower QOL score, recommending that the results of vertebral fracture are related to QOL (23). Also, regarding to the findings, $\mathrm{MH}$ was most effective domain, which significantly influenced the total score of HR-QOL. By contract, Dennison et al. demonstrated that in osteoporotic women, MH, SF and RE are related with total femur bone density (19). Also, the MH field is that most of the other areas are affected by confounding factors and it is important to consider all characteristics before interpreting the changes (19). Moreover, MH was considered as major domain of HR-QOL compared to other domains for predicting the QOL worsening in progression of bone density loss (24).

Moreover, femoral neck BMD changes were significantly correlated with BMI and weight and in the other hand physical activity and somatic-related domain of HR-QOL such as BP and RP were correlated with BMI; however, they were not significantly correlated with femoral BMD score changes. According to our findings, previous studies confirmed that exercise provide modest benefits for post-menopaus women with OPN such as increasing in BMD and also improved the HR-QOL of these patients (25). Overall, it can be assumed that mental health-related QOL are more likely related with spinal BMD changes and physical health-related QOL domains are more likely related with femoral neck BMD changes. In contrast, Dennison et al. demonstrated that they have not found any relationships between the lumbar spine bone loss rate and HR-QOL in any domain.

Previously, calcitonin has been certified for treatment of symptomatic OPS although the recent studies clarified the efficacy of nasal form of calcitonin $(26,27)$. In this study bodily pain and physical role functioning (PR) had significant changes over the 6-month treatment period with calcitonin, which these findings are consistent with each other. Level of education was identified as an effective factor on the progression of OPS and response to the treatment according to the BMD improvement and in this study, educational status was correlated with HRQOL changes (28). Education can enhance the patients' insight and improve the life style and thus assist to the plan of therapy. Also, in the present study, we found that BMD T-score of femoral neck was significantly associated with weight and BMI, which it is relatively supported by the previous studies $(23,28)$.

The limitation of this study was short-term duration of follow-up and we have not observed long-term effects of nasal calcitonin on HR-QOL. Moreover, we did not have a control group to exclude the role of race or nationality although no patients had past history of psychiatric disorders. Finally, to confirm the analgesic role of nasal calcitonin on the back pain and also QOL in nonfractured OPN, we recommend using a quantitative scale such as Visual Analogue Scale (VAS) along with the QOL questionnaire.

According to the results of this study, it seems intranasal calcitonin can effectively improve QOL of women with low bone density via improving in bone density and BMD and also decreasing bone turnover. Furthermore, it seems that HR-QOL changes were not influenced by demographic or clinical characteristics.

\section{Acknowledgements}

The authors would like to thank all participants for their kindly cooperation.

\section{Authors' Contributions}

Majid Shohrati, Noushin Bayat and Amin Saburi design the study and develop the idea. Majid Shohrati, Noushin Bayat, Zahra Abbasi gartered the data. Amin Saburi analyzed the data. Majid Shohrati, Amin Saburi and Noushin Bayat interpreted the findings and prepared the initial draft. All authors approved the final draft.

\section{Funding/Support}

This project was supported by Sinadaru Company and the chemical injury research center of Baqiyatallah University of Medical Sciences.

\section{References}

1. Genant HK, Cooper C, Poor G, Reid I, Ehrlich G, Kanis J, et al Interim report and recommendations of the World Health Organization Task-Force for Osteoporosis. Osteoporos Int. 1999;10(4):259-64

2. Ofluoglu D, Karadag-Saygi E, Canbulat C, Gunduz OH, Kul-Panza E, Akyuz G. Early effect of nasal salmon calcitonin on the bone marker Crosslaps. Rheumatol Int. 2006;26(4):288-91.

3. Hallberg I, Rosenqvist AM, Kartous L, Lofman O, Wahlstrom O, Toss G. Health-related quality of life after osteoporotic fractures. Osteoporos Int. 2004;15(10):834-41.

4. Weiss M, Ben-Shlomo AB, Hagag P, Rapoport M. Reference database for bone speed of sound measurement by a novel quantitative multi-site ultrasound device. Osteoporos Int. 2000;11(8):688-96.

5. Chesnut CH, Silverman S, Andriano K, Genant H, Gimona A, Harris $\mathrm{S}$, et al. A randomized trial of nasal spray salmon calcitonin in postmenopausal women with established osteoporosis: the prevent recurrence of osteoporotic fractures study. The American Journal of Medicine. 2000;109(4):267-76.

6. Huusko TM, Karppi P, Kautiainen H, Suominen H, Avikainen V, 
Sulkava R. Randomized, double-blind, clinically controlled trial of intranasal calcitonin treatment in patients with hip fracture. CalcifTissue Int. 2002;71(6):478-84.

7. Trovas GP, Lyritis GP, Galanos A, Raptou P, Constantelou E. A randomized trial of nasal spray salmon calcitonin in men with idiopathic osteoporosis: effects on bone mineral density and bone markers. J of Bone and Mineral Res. 2002;17(3):521-7.

8. Ellerington MC, Hillard TC, Whitcroft SI, Marsh MS, Lees B, Banks LM, et al. Intranasal salmon calcitonin for the prevention and treatment of postmenopausal osteoporosis. Calcif Tissue Int. 1996;59(1):6-11.

9. Kaskani E, Lyritis GP, Kosmidis C, Galanos A, Andypas G, Chorianopoulos $\mathrm{K}$, et al. Effect of intermittent administration of 200 IU intranasal salmon calcitonin and low doses of 1 alpha $(\mathrm{OH})$ vitamin D3 on bone mineral density of the lumbar spine and hip region and biochemical bone markers in women with postmenopausal osteoporosis: a pilot study. Clin Rheumatol. 2005;24(3):232-8.

10. Montazeri A, Goshtasebi A, Vahdaninia M, Gandek B. The Short Form Health Survey (SF-36): translation and validation study of the Iranian version. Qual Life Res. 2005;14(3):875-82.

11. Motamed N, Ayatollahi AR, Zare N, Sadeghi-Hassanabadi A. Validity and reliability of the Persian translation of the SF-36 version 2 questionnaire. East Mediterr Health J. 2005;11(3):349-57.

12. Ali-Mohammadpour R, Yousefi Z. Factor analysis of SF-36 Persian version health-related quality of life questionnaire in Iran. World Applied Sciences Journal. 2008;3(4):548-54.

13. Persson LO, Karlsson J, Bengtsson C, Steen B, Sullivan M. The Swedish SF-36 Health Survey II. Evaluation of clinical validity: results from population studies of elderly and women in Gothenborg.J Clin Epidemiol. 1998;51(11):1095-103.

14. Sullivan M, Karlsson J. The Swedish SF-36 Health Survey III. Evaluation of criterion-based validity: results from normative population. JClin Epidemiol.1998;51(11):1105-13.

15. Sullivan M, Karlsson J, Ware JE. The Swedish SF-36 Health SurveyI. Evaluation of data quality, scaling assumptions, reliability and construct validity across general populations in Sweden. Social Science \& Medicine. 1995;41(10):1349-58.

16. Brown JP, Josse RG, Scientific Advisory Council of the Osteoporosis Society of C. 2002 clinical practice guidelines for the diagnosis and management of osteoporosis in Canada. CMAJ. 2002;167(10 Suppl):S1-34.

17. Sanchez-Riera L, Wilson N, Kamalaraj N, Nolla JM, Kok C, Li Y, et al.
Osteoporosis and fragility fractures. Best Pract Res Clin Rheumatol. 2010;24(6):793-810.

18. Bliuc D, Nguyen ND, Milch VE, Nguyen TV, Eisman JA, Center JR. Mortality risk associated with low-trauma osteoporotic fracture and subsequent fracture in men and women. JAMA. 2009;301(5):513-21.

19. Dennison EM, Jameson KA, Syddall HE, Martin HJ, Cushnaghan J, Aihie Sayer A, et al. Bone health and deterioration in quality of life among participants from the Hertfordshire cohort study. Osteoporos Int. 2010;21(11):1817-24.

20. Lyritis GP, Ioannidis GV, Karachalios T, Roidis N, Kataxaki E, Papaioannou N, et al. Analgesic effect of salmon calcitonin suppositories in patients with acute pain due to recent osteoporotic vertebral crush fractures: a prospective double-blind, randomized, placebo-controlled clinical study. Clin J Pain. 1999;15(4):284-9.

21. Ofluoglu D, Akyuz G, Unay O, Kayhan O. The effect of calcitonin on beta-endorphin levels in postmenopausal osteoporotic patients with back pain. Clin Rheumatol. 2007;26(1):44-9.

22. Delmas PD. Markers of bone turnover for monitoring treatment of osteoporosis with antiresorptive drugs. Osteoporosis international. 2000;11(18):S66-76.

23. Yoshimura N, Kinoshita H, Takijiri T, Oka H, Muraki S, Mabuchi A, et al. Association between height loss and bone loss, cumulative incidence of vertebral fractures and future quality of life: the Miyama study. Osteoporosis International. 2008;19(1):21-8.

24. Romagnoli E, Carnevale V, Nofroni I, D’Erasmo E, Paglia F, De Geronimo S, et al. Quality of life in ambulatory postmenopausal women: the impact of reduced bone mineral density and subclinical vertebral fractures. Osteoporosis international. 2004;15(12):975-80.

25. Bolton KL, Egerton T, Wark J, Wee E, Matthews B, Kelly A, et al. Effects of exercise on bone density and falls risk factors in postmenopausal women with osteopenia: a randomised controlled trial.J Sci Med Sport. 2012;15(2):102-9.

26. Mehta NM, Malootian A, Gilligan JP. Calcitonin for osteoporosis and bone pain. Curr Pharm Des. 2003;9(32):2659-76.

27. Gennari C, Agnusdei D, Camporeale A. Use of calcitonin in the treatment of bone pain associated with osteoporosis. Calcif Tissue Int. 1991;49 Suppl 2:S9-13.

28. Gemalmaz A, Discigil G, Sensoy N, Basak O. Identifying osteoporosis in a primary care setting with quantitative ultrasound: relationship to anthropometric and lifestyle factors. J Bone Miner Metab. 2007;25(3):184-92. 